BULL. AUSTRAL. MATH. SOC.

\title{
INTERSECTIONS OF FINITELY GENERATED \\ FREE GROUPS
}

\section{Peter Nickolas}

A result of Howson is that two finitely generated subgroups $U$ and $V$ of a free group have finitely generated intersection. Hanna Neumann showed further that, if $m, n$ and $N$ are the ranks of $U, V$ and $U \cap V$ respectively, then $N \leq 2(m-1)(n-1)+1$, and Burns strengthened this, showing that $N \leq 2(m-1)(n-1)-m+2$ (if $m \leq n$ ). This paper presents a new and simple proof of Burns' result. Further, the graph-theoretical ideas used provide still stronger bounds in certain special cases.

\section{Introduction}

Let $U$ and $V$ be subgroups of a free group $F$, with $U$ and $V$ of finite ranks $m$ and $n$ respectively. Howson [4] showed that $U \cap V$ is also finitely generated, and gave a bound on its rank $N$ in terms of $m$ and $n$. Neumann $[7,8]$ subsequently sharpened Howson's bound, showing that $N \leq 2(m-1)(n-1)+1$. On the other hand, if $U$ or $V$ is of finite index in $F$, then the stronger bound $N \leq(m-1)(n-1)+1$ holds (Burns [1]), and most writers on the subject seem to believe that this bound should hold in all cases. (Howson [4] has examples showing that no bound lower than this is possible.)

The strongest bound known to hold in general, however, is due to Burns

Received 31 October 1984.

Copyright Clearance Centre, Inc. Serial-fee code: 0004-9727/85 $\$ 12.00+0.00$. 
[2], who shows that $N \leq 2(m-1)(n-1)-m+2$ (taking $m \leq n$ ). Our purpose in this paper is to examine the problem again, obtaining some detailed information ( $(2)$ from which Burns' bound in particular follows.

We approach the problem graph-theoretically, as do most other writers on the subject [3]-[10]. (Servatius [9] has recently shown how Burns' argument in [2] may be rewritten graph-theoretically.) Our main argument takes the form of a simple graph-theoretical algorithm. The information obtained from this not only yields Burns' result, but also enables us to obtain better bounds in two interesting special cases. The first of these is when $m=2$ : here the presumed optimal bound $N \leq(m-1)(n-1)+1$ reduces simply to $N \leq n$ and we are able to show that, for a given $U$ of rank 2 , this bound holds for (in some sense) most subgroups $V$. The second special case considered is when $V$ is a conjugate of $U$ : here, of course, $m=n$, and we show that $N \leq 2(m-1)^{2}-3 m+5$.

The reader will find it useful to have some familiarity with the use of graphs in combinatorial group theory, as, for example, in [5].

\section{A graph-theoretical algorithm}

2.1. We assume without loss of generality that $U$ and $V$ are subgroups of $F=F(a, b)$, the free group on generators $a$ and $b$. For any finitely generated subgroup, say $U$, of $F$, a standard construction [6] yields a finite graph $\Gamma(U)$ as follows. First let $\Gamma_{U}$ be the graph with vertices the distinct cosets $U x \quad(x \in F)$, and edges all pairs $\left(U_{x}, y\right)$, for $x \in F$ and $y \in\{a, b\}$, and where $(U x, y)$ runs from $U x$ to Uxy . (Of course, $\Gamma_{U}$ is a quotient graph of the Cayley graph [5] of $F$ with respect to $\{a, b\}$.$) Then \Gamma(U)$ is the smallest subgraph of $\Gamma_{U}$ containing all cycles; that is (following [10]), the core of $\Gamma_{U}$. Since $U$ is finitely generated, $\Gamma(U)$ is finite. (Note that $\Gamma(U)$ need not contain the vertex $U$.$) Informally, we think of \Gamma(U)$ as a directed, labelled graph with labels chosen from $\{a, b\}$. In particular we may represent $\Gamma(F)$ as




Clearly, all vertices of $\Gamma(U)$ are of degree 2,3 or 4 and, as in [9], we may suppose them to be of degrees 2 and 3 only. (Isomorphically embed $F$ into $F(\alpha, \beta)$ via the map $a \mapsto \alpha B^{-1}$, $b \mapsto \beta^{2}$, and take graphs relative to the new generators $\left.\alpha, \beta.\right)$ We refer to vertices of degree 3 as branch points.

2.2. The crucial fact now is that $\Gamma(U \cap V)$ may be identified with the core of a connected component of the graph $\Gamma(U) \tilde{x} \Gamma(V)$, obtained as a pullback in the diagram

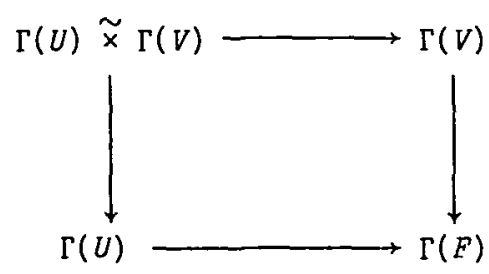

in the category of directed, $\{a, b\}$-labelled graphs, and where the maps into $\Gamma(F)$ are the obvious projections. (We still think of $\Gamma(F)$ in the form drawn in 2.1, though $\Gamma(U)$ and $\Gamma(V)$ now have no vertices of degree 4.)

In a graph such as $\Gamma(U)$, with vertices of degrees 2 and 3 only, we see easily that the cyclomatic number is 1 plus one-half the number of branch points. Since the rank of the subgroup is the cyclomatic number of its graph, to obtain bounds on the rank $N$ of $U \cap V$, we need to bound the number of branch points in the core of $\Gamma(U) \widetilde{x} \Gamma(V)$.

2.3. There are four possible labellings of the edges incident with a branch point, as follows:
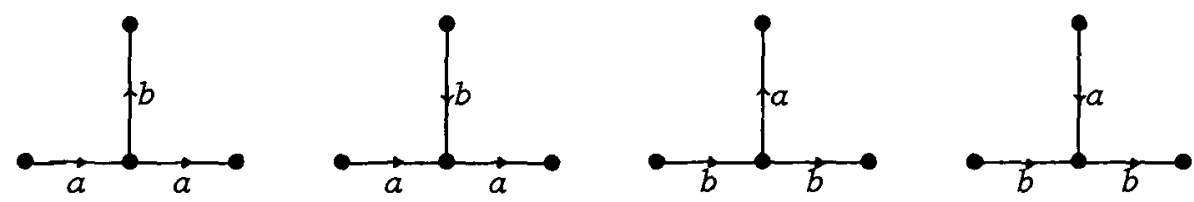

We refer to vertices so labelled, respectively, as $b$-sources, b-sinks, a-sources, and a-sinks. (Here of course a loop contributes an inwardand an outward-going edge.)

2.4. Consider any two branch points $X$ in $\Gamma(U)$ and $Y$ in $\Gamma(V)$. There are exactly three (reduced) paths $p_{1}, p_{2}, p_{3}$ beginning at $X$ and 
ending at the first branch point encountered; and $q_{1}, q_{2}, q_{3}$ are similarly defined at $Y$. There is also a natural pairing of each $p_{i}$ with some $q_{j}$; for, at least two of the labels of the edges at $X$ are equal to those of edges at $Y$; we pair those paths beginning with similarly labelled edges, and then, if two remain unpaired we pair them. Suppose for convenience that $p_{i}$ is paired with $q_{i}, i=1,2,3$. Then we say that $p_{i}$ and $q_{i}$ are compatible if there is a path $p$ in $\Gamma(U) \tilde{x} \Gamma(V)$ from $(X, Y)$ to a vertex $\left(X^{\prime}, Y^{\prime}\right)$, where $X^{\prime}$ and $Y^{\prime}$ are branch points in $\Gamma(U)$ and $\Gamma(V)$ respectively, and where the projections of $p_{i}$ and $q_{i}$ into $\Gamma(F)$ are both initial segments of the projection of $p$. The branch points $X$ and $Y$ are compatible if $p_{i}$ and $q_{i}$ are compatible for each $i$. (Incompatible, of course, will mean not compatible.)

Now it is easy to see that whenever $(X, Y)$ is a branch point in the core of $\Gamma(U) \widetilde{\times} \Gamma(V), X$ and $Y$ must be compatible. Since, as mentioned in 2.2, our concern is to bound the number of branch points in the core, it therefore suffices to bound the number of such compatible pairs. Our main result, which we now state, enables us to do this; its proof occupies the remainder of $\S 2$.

THEOREM 2.5. Given $U, V \leq F$ of ranks at least 2 , there is a partition of the branch points of $\Gamma(U) \cup \Gamma(V)$ (where $\Gamma(U)$ and $\Gamma(V)$ are taken as disjoint) into two non-empty sets $P_{1}$ and $P_{2}$, with the following property: all branch points of $P_{1} \cap \Gamma(U)$ are incompatible with those of $P_{2} \cap \Gamma(V)$, and all branch points of $P_{2} \cap \Gamma(U)$ are incompatible with those of $P_{1} \cap \Gamma(V)$.

2.6. If among the branch points of $\Gamma(U) \cup \Gamma(V)$ two or more distinct labelling types occur (2.3), put all vertices of one fixed type in $P_{1}$, and the others in $P_{2}$ : the conclusion of the theorem clearly holds.

2.7. Otherwise, suppose that all labelling types of branch points in $\Gamma(U) \cup \Gamma(V)$ are the same. For concreteness, let us assume all branch points to be $b$-sources (2.3). We lose no generality by doing so, since 
any type of branch point can be converted into a b-source by systematic relabelling; that is, performing throughout $\Gamma(U)$ and $\Gamma(V)$ a suitably chosen sequence of the following steps:

replace all $a$ labels by $b$ labels, and vice versa;

reverse all edges labelled $a$;

reverse all edges labelled $b$.

2.8. Let $\Gamma_{0}=\Gamma(U)$ and $\Delta_{0}=\Gamma(V)$, so that $\Gamma_{0}$ and $\Delta_{0}$ are directed, $\{a, b\}$-labelled graphs, with vertices of degree 2 and 3 only, and with all branch points being $b$-sources. Additionally, because of the group-theoretical origin of $\Gamma_{0}$ and $\Delta_{0}$, no paths of the forms
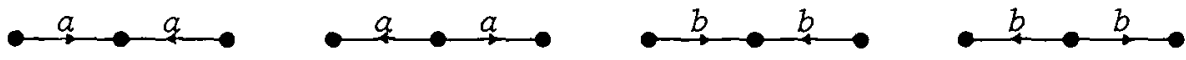

ever occur.

We now describe an algorithm which, from the pair $\left(\Gamma_{0}, \Delta_{0}\right)$, successively produces pairs $\left(\Gamma_{1}, \Delta_{1}\right), \ldots$ of graphs of exactly the same type, except that, for some $k, \Gamma_{k}$ and $\Delta_{k}$ will contain branch points of two distinct kinds, at which point the algorithm will terminate.

2.9. The operation which transforms $\Gamma_{i-1}$ and $\Delta_{i-1}$ into $\Gamma_{i}$ and $\Delta_{i}$ is accomplished in the following steps, governed by the rule given below.

(1) In $\Gamma_{i-1}$ and $\Delta_{i-1}$ replace all (maximal) paths of the form

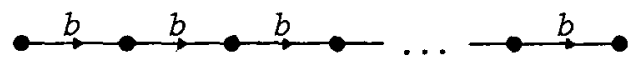

by

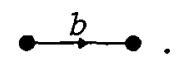

(2) If paths of the form them all by (3). Otherwise, go to step (4).

(3) Repeat step (2).

(4) If paths of the form




all by $\longrightarrow$, and invoke the rule.

(5) Set $\Gamma_{i}$ and $\Delta_{i}$ equal to the transformed graphs.

RULE. If all branch points are $b$-sources, take no action. If all branch points are of some one fixed type, but are not $b$-sources, relabel the graphs (2.7) and restart the algorithm at step (1).

If two distinct branch point labelling types occur, place all branch points of one type in $P_{1}$ and all the others in $P_{2}$, set $\Gamma_{i}$ and $\Delta_{i}$ equal to the transformed graphs, and stop.

2.10. We make the following assertions, giving an indication of the proofs in parentheses.

(1) The algorithm is well-defined. (Distinct paths chosen for contraction in any one step are edge-disjoint, so that the instruction to contract such paths is unambiguous; and these paths never pass through branch points (except perhaps at their ends), so that the action of replacing them by $\stackrel{b}{\longrightarrow}$ is well-defined.)

(2) $\Gamma_{i}$ and $\Delta_{i}$ are of exactly the same type (2.8) as $\Gamma_{i-1}$ and $\Delta_{i-1}$ (with the possible exception of branch point labellings). (The only point requiring checking is that paths such as $\stackrel{\longrightarrow}{\longrightarrow}$ and so on cannot appear. Step (2), for example, could only produce such a path (necessarily of the form $\longrightarrow b \_b-b$ ) if a contracted path $\bullet \stackrel{b}{a} \rightarrow a b$ were contained in a longer path $\stackrel{b}{\longrightarrow} a \bullet b \bullet b \bullet$, and this is impossible, as paths such as $b \longrightarrow b \longrightarrow$ have been eliminated by step (1). Similar arguments apply to the other contractions.)

(3) The numbers of branch points in $\Gamma_{i-1}$ and $\Delta_{i-1}$ are exactly preserved in an application of the algorithm. (Obvious.)

(4) The number of branch points in the core of $\Gamma_{i} \tilde{\times} \Delta_{i}$ is at least 
as large as the number in the core of $\Gamma_{i-1} \widetilde{\times} \Delta_{i-1}$. (This follows from the obvious fact that compatibility of paired paths is preserved by the algorithm, and from the relationship between branch points and compatibility (see 2.4).)

(5) The algorithm terminates, and does so only when two types of labelling appear. (Since we strictly decrease the number of edges with each application of the algorithm, the algorithm must stop. It will do so if two types of labelling appear, or if no paths exist of the kinds contracted by the algorithm. The latter is easily seen to be impossible, given that all branch points are $b$-sources.)

(6) $P_{1}$ and $P_{2}$ (strictly, the corresponding sets of vertices in $\Gamma(U)$ and $\Gamma(V)$ ) are as claimed in the theorem. (This follows from the fact ((4) above) that compatibility is preserved.)

The theorem is now proved.

\section{Applications}

3.1. Using the notation of the theorem, let $u_{i}$ and $v_{i}$ be the cardinalities of $P_{i} \cap \Gamma(U)$ and $P_{i} \cap \Gamma(V)$ respectively, for $i=1,2$. Then from the theorem, and the relation (2.4) between branch points and compatibility, we have the

COROLLARY. In the core of $\Gamma(U) \widetilde{x} \Gamma(V)$ there are at most $u_{1} v_{1}+u_{2} v_{2}$ branch points.

THEOREM 3.2 (Burns [2]). If $U, V \leq F$ have ranks $m, n$ respectively, and $1 \leq m \leq n$, then the rank $N$ of $U \cap V$ satisfies

$$
N \leq 2(m-1)(n-1)-m+2 \text {. }
$$

Proof. The result is trivial if $m=1$, so let $m \geq 2$. By 2.2, $\Gamma(U)$ and $\Gamma(V)$ have $2(m-1)$ and $2(n-1)$ branch points respectively, so, in the notation of the corollary, $u_{1}+u_{2}=2(m-1)$ and $v_{1}+v_{2}=2(n-1)$ Therefore $u_{1} v_{1}+u_{2} v_{2}=4(m-1)(n-1)-\left(u_{2} v_{1}+u_{1} v_{2}\right)$. But from the fact that the sets $P_{1}$ and $P_{2}$ given by the main theorem are non-empty, we se $\epsilon$ easily that $u_{2} v_{1}+u_{1} v_{2} \geq 2(m-1)$, and so 
$u_{1} v_{1}+u_{2} v_{2} \leq 4(m-1)(n-1)-2(m-1)$, from which the desired result follows by 2.2 and the corollary.

3.3. The first of the special cases mentioned in the introduction is where the subgroup $U$ has rank $m=2$, and $\Gamma(U)$ contains just two branch points.

First, consider the application of the algorithm to two disjoint copies of $\Gamma(U)$. Denote the branch points in the first copy of $\Gamma(U)$ by $X$ and $Y$, and the corresponding branch points in the second copy by $X^{\prime}$ and $Y^{\prime}$. Then it is easy to see that one of the sets $P_{1}, P_{2}$ produced by the algorithm must contain $X$ and $X^{\prime}$, and the other $Y$ and $Y^{\prime}$. From 3.1 we therefore see that the core of $\Gamma(U) \widetilde{x} \Gamma(U)$ contains at most two branch points; and since the component of the core containing $\left(X, X^{\prime}\right)$ certainly has two branch points (corresponding to the fact that $U \cap U=U$ ), the number is exactly two.

In particular, $\left(X, Y^{\prime}\right)$ and $\left(X^{\prime}, Y\right)$ have degree at most 2 in the core, and hence there exist edges $x_{1}$ at $X$ and $y_{1}$ at $Y$ (or, strictly, $Y^{\prime}$ ), belonging to paired paths (2.4), and such that no path in the core at $\left(X, Y^{\prime}\right)$ commences with the edge $\left(x_{1}, y_{1}\right)$.

LEMMA. There is a largest integer $l \geq 0$ for which there are reduced paths $x_{1} x_{2} \ldots x_{2}$ and $y_{1} y_{2} \ldots y_{2}$ at $x$ and $y$ (respectively), having equal projections in $\Gamma(F)$.

Proof. If no such largest $l$ exists, there is a path $\left(x_{1}, y_{1}\right)\left(x_{2}, y_{2}\right) \ldots$ in $\Gamma(U) \tilde{\times} \Gamma(U)$ which may be extended to any length. Since $\Gamma(U) \widetilde{x} \Gamma(U)$ is finite, this path must contain a cycle. If the cycle contains $\left(X, Y^{\prime}\right)$, then there is a path in the core containing $\left(X, Y^{\prime}\right)$ and commencing with $\left(x_{1}, y_{1}\right)$, a contradiction. If $\left(X, Y^{\prime}\right)$ is not in the cycle, the cycle contains a branch point, which must clearly be $\left(X^{\prime}, Y\right)$. By symmetry, there is also a cycle containing $\left(X, Y^{\prime}\right)$ and not $\left(X^{\prime}, Y\right)$, and since a path starting with $\left(x_{1}, y_{1}\right)$ joins the two cycles, this path is in the core, also giving a contradiction. Thus the integer $\tau$ exists as required.

It is now clear, given another subgroup $V$, that any path in $\Gamma(V)$ 
of length greater than 2 , paired with the paths $p$ and $q$ containing $x_{1}$ and $y_{1}$, can be compatible with at most one of $p$ and $q$. Therefore we have the

PROPOSITION. Let $V$ have rank $n \geq 2$. If in $\Gamma(V)$ all paths paired with $p$ and $q$ are of length greater than $l$, then $N \leq n$.

3.4. The second special case is as follows.

PROPOSITION. Let $U$ be of rank $m>2$. Then for any $x \in F$, the rank $N$ of $U \cap x^{-1} U x$ satisfies

$$
N \leq 2(m-1)^{2}-3 m+5 \text {. }
$$

Proof. Writing $V=x^{-1} U x$, we see easily that $\Gamma(U)$ and $\Gamma(V)$ are isomorphic. In an application of the algorithm to $\Gamma(U)$ and $\Gamma(V)$, therefore, any changes made to $\Gamma(U)$ are made simultaneously to $\Gamma(V)$, and vice versa. It follows that (in the notation of the corollary) $u_{1}=v_{1}$ and $u_{2}=v_{2}$. Then, from the fact that the sets $P_{1}$ and $P_{2}$ produced by the algorithm are non-empty, and since $\Gamma(U)$ and $\Gamma(V)$ have $2(m-1)$ branch points (2.2), we see easily that $u_{1} v_{1}+u_{2} v_{2}$ can be no greater than $1.1+(2 m-3)(2 m-3)=4 m^{2}-12 m+10$. Hence, by the corollary, the core of $\Gamma(U) \widetilde{x} \Gamma(V)$ contains at most this number of branch points. However, the component of the core corresponding to the intersection of $U$ with itself already contains exactly $2(m-1)$ branch points, so any other component can have at most $4 m^{2}-14 m+12$ branch points. The ranks of the intersections represented by such a component must therefore be bounded as stated, by 2.2 , and since also $m \leq 2(m-1)^{2}-3 m+5$ when $m>2$, the result follows.

\section{References}

[1] R.G. Burns, "A note on free groups", Proc. Amer. Math. Soc. 23 (1969), 14-17.

[2] Robert G. Burns, "On the intersection of finitely generated subgroups of a free group", Math. 2. 119 (1971), 121-130. 
[3] S.M. Gersten, "Intersections of finitely generated subgroups of free groups and resolutions of graphs", Invent. Math. 71 (1983), 567-591.

[4] A.G. Howson, "On the intersection of finitely generated free groups", J. London Math. Soc. 29 (1954), 428-434.

[5] Wilfried Imrich, "Subgroup theorems and graphs", Combinatorial Mathematics $V, \quad 1-27$ (Lecture Notes in Mathematics, 622. Springer-Verlag, Berlin, Heidelberg, New York, 1977).

[6] Wilfried Imrich, "On finitely generated subgroups of free groups", Arch. Math. 28 (1977), 21-24.

[7] Hanna Neumann, "On the intersection of finitely generated free groups", Publ. Math. Debrecen 4 (1955-56), 186-189.

[8] Hanna Neumann, "On the intersection of finitely generated free groups: Addendum", Publ. Math. Debrecen 5 (1957-58), 128.

[9] Brigitte Servatius, "A short proof of a theorem of Burns", Math. Z. 184 (1983), 133-137.

[10] John R. Stallings, "Topology of finite graphs", Invent. Math. 71 (1983), 551-565.

Department of Mathematics, University of Queensland,

St Lucia,

Queensland 4067,

Australia. 